\title{
EFEITO DA APLICAÇÃO DE PÓ DE ROCHA FOSFATADA NO DESENVOLVIMENTO INICIAL DE MUDAS DE Schizolobium parahyba var. amazonicum (Huber ex Ducke) Barneby.
}

Effect of phosphated rock powder application on the initial development of Schizolobium parahyba var. amazonicum (Huber ex Ducke) Barneby.

Efecto de la aplicación de polvo de roca fosfatado en el desarrollo inicial de Schizolobium parahyba var. amazonicum (Huber ex Ducke) Barneby.

Bruno Aurélio Campos Aguiar*1, Hygor Gomes de Almeida Sousa ${ }^{2}$, Eduardo Moraes de Oliveira $^{3}$, Marcos Vinicius de Melo Amorim ${ }^{4}$, Millena Oliveira Xavier ${ }^{5}$, Priscila Bezerra de Souza ${ }^{6}$

${ }^{1}$ Laboratório de Sementes Florestais, Universidade Federal do Tocantins, campus de Gurupi.

*Correspondência: Laboratório de Sementes Florestais, Universidade Federal do Tocantins, Rua Badejos, Lote 7 s/n-Jardim Sevilha, Gurupi - TO, 77404-970. E-mail aguiar.florestal@ gmail.com.

\section{Artigo recebido em 16/08/2019 aprovado em 29/10/2019 publicado em 23/03/2020 .}

\section{RESUMO}

Objetivou-se avaliar os efeitos da aplicação do pó de rocha fosfatada como substrato na 3 de mudas de Paricá (Shizolobium amazonicum). O experimento foi desenvolvido no viveiro Florestal da Universidade Federal do Tocantins, campus de Gurupi, sendo conduzido em delineamento inteiramente casualizado, constituído por 5 tratamentos: T1: $0 \%$ de pó de rocha (PDR) + 100\% de Substrato comercial; T2: $25 \%$ de PDR + 75\% de Substrato comercial; T3: 50\% de PDR + 50\% de Substrato comercial; T4: 75\% de PDR + 25\% de Substrato comercial; T5: 100\% de PDR + 0\% de Substrato comercial), com 4 repetições, onde em cada repetição foram avaliadas as médias de 10 plântulas. Foram observados os efeitos da adição do pó de rocha ao substrato comercial no desenvolvimento da espécie Shizolobium amazonicum. As mudas de Shizolobium amazonicum produzidas com baixas proporções de pó de rocha ao substrato comercial tiveram uma boa resposta (Efeito positivo) no seu desenvolvimento. Portanto recomenda-se utilização de uma mistura de $25 \%$ PDR + 75\% substrato comercial, elevando os níveis minerais do substrato, pois esse tratamento permitiu melhor desempenho germinativo, mostrando-se mais adequados para a produção eficaz de mudas da espécie.

Palavras-chave: Paricá. Sementes Florestais. Substrato.

\section{ABSTRACT}

The objective of this study was to evaluate the effects of phosphate rock powder application as substrate in the production of Paricá (Shizolobium amazonicum) seedlings. The experiment was carried out at the Forest Nursery of the Federal University of Tocantins, Gurupi campus, and was conducted in a completely randomized design consisting of 5 treatments: T1: 0\% rock dust $(P D R)+100 \%$ commercial substrate; T2: $25 \%$ PDR + 75\% Commercial Substrate; T3: 50\% PDR + 50\% Commercial Substrate; T4: 75\% PDR + 25\% Commercial Substrate; T5: 100\% PDR + 0\% Commercial Substrate), with 4 replications, where in each repetition the averages of 10 
seedlings were evaluated. The effects of the addition of rock dust to the commercial substrate on the development of Shizolobium amazonicum species were observed. Shizolobium amazonicum seedlings produced with low proportions of rock dust to the commercial substrate had a good response (positive effect) on their development. Therefore, it is recommended to use a mixture of $25 \%$ PDR $+75 \%$ commercial substrate, increasing the substrate mineral levels, as this treatment allowed better germination performance, being more suitable for the effective production of seedlings of the species.: Paricá. Forest seeds. Substrate.

Keywords: Paricá. Forest seeds. Substrate.

\section{RESUMEN}

El objetivo de este estudio fue evaluar los efectos de la aplicación de polvo de roca de fosfato como sustrato en la producción de plántulas de Paricá (Shizolobium amazonicum). El experimento se llevó a cabo en el vivero forestal de la Universidad Federal de Tocantins, campus de Gurupi, y se realizó en un diseño completamente al azar que consta de 5 tratamientos: T1: $0 \%$ de polvo de roca $(P D R)+100 \%$ de sustrato comercial; T2: $25 \%$ de PDR + 75\% de sustrato comercial; T3: 50\% de PDR + 50\% de sustrato comercial; T4: 75\% de PDR + 25\% de sustrato comercial; T5: 100\% PDR + 0\% de sustrato comercial), con 4 repeticiones, donde en cada repetición se evaluaron los promedios de 10 plántulas. Se observaron los efectos de la adición de polvo de roca al sustrato comercial en el desarrollo de especies de Shizolobium amazonicum. Las plántulas de Shizolobium amazonicum producidas con bajas proporciones de polvo de roca al sustrato comercial tuvieron una buena respuesta (efecto positivo) en su desarrollo. Por lo tanto, se recomienda usar una mezcla de $25 \%$ de PDR $+75 \%$ de sustrato comercial, aumentando los niveles minerales del sustrato, ya que este tratamiento permitió un mejor rendimiento de germinación, siendo más adecuado para la producción efectiva de plántulas de la especie.

Descriptores: Paricá. Semillas del bosque. Sustrato.

\section{INTRODUÇÃO}

SChizolobium parahyba var. amazonicum (Huber exDucke) Barneby. tem ocorrência principalmente na Bacia Amazônica e sua distribuição está relacionada a mata primária de terra firme, várzea alta e florestas secundárias, podendo formar em algumas regiões capoeiras com grande dominância monoespecífica (ABRAF, 2013).

É uma espécie essencialmente heliófila, que não tolera baixas temperaturas, apresenta crescimento monopodial, ainda que a céu aberto, com fuste reto e limpo, devido à boa desrama natural ou auto-poda (DIAS et al., 2015).
De acordo com Iwakiri et al. (2010), a madeira pode ser utilizada na produção de lâminas médias ou miolo de compensados, brinquedos, caixotaria leve, portas e parquete, energia com a produção de lenha de qualidade razoável, na indústria de celulose e papel. O Paricá é uma espécie promissora para a produção de pasta para celulose, destacando-se seu fácil branqueamento e as excelentes resistências obtidas com o papel branqueado (IWAKIRI et al., 2010).

Além da sua utilização nos plantios para recuperação, ou seja, a espécie é recomendada para restauração de ambientes ripários em locais não sujeitos a inundação, com crescimento rápido, apresenta incrementos 
em altura e diâmetro capazes de possibilitar sua exploração já aos 15 anos de idade (THOMAZ, 2012). Entretanto, o substrato é de suma importância para se produzir mudas de qualidade, pois suas propriedades físicoquímicas devem proporcionar o desenvolvimento da muda.

Por causa da pequena capacidade do recipiente e a constante lixiviação de nutrientes ocorrido durante as irrigações de viveiro, é necessário realizar a adubação de cobertura nas mudas (MAEDA et al., 2007). Essa exigência de nutrientes aumenta o custo, no entanto, instiga novos estudos com produtos alternativos para adubação (KNAPIK; ÂNGELO, 2007) e para substratos. De acordo com Stappe e Balloni (1988), os insumos não convencionais como pó de rochas Gnaisse, tem se tornado atraentes por ter baixo valor e aprimorar as propriedades de solo e elevar a produção vegetal. Para Theodoro (2006), o mineral citado anteriormente, apresenta grandes quantidades de macronutrientes, podendo ser agregado a materiais orgânicos, tornando-se alternativa de insumo, desde que esteja presente na região e não apresente contaminantes.

Rochas Gnaisse detém minerais intemperizáveis e ricos em cátions, como feldspato potássico, plagioclásio, e ainda quartzo e biota (RESENDE et al., 2002), maximizando o uso de seu pó em substratos. A facilidade para consegui-lo em pedreiras vulcânicas por todo Brasil diminui o seu custo em relação a outros produtos para a adubação (KIEHL, 2002). Para Theodoro (2000), isso propicia o seu uso em solos com custo quase 20 vezes menor do que o de insumos usuais.
Estudos relacionados ao efeito do pó de rocha na microbiota do solo e de seu agrupamento indicam o quão pode ser importante o pó de rocha fosfatada para uso em solos (SUGUINO et al., 2011) e para fazer parte em substratos para mudas.

Nesse contexto, o pó de rocha é um resíduo resultante das operações de mineração, que apresenta valor comercial muito baixo, tornando sua comercialização inviável. No entanto, o uso de pó de rocha em clima tropical tem grande potencialidade, pois as taxas de dissolução dos minerais e as reações entre a superfície dos minerais e a solução do solo são favorecidas sob condição de temperatura elevada e alta umidade relativa, além de auxiliar na redução dos altos custos gerados pelos fertilizantes sintéticos (WOLSCHICK et al., 2016).

Diante de poucas informações a respeito do uso de pó de rocha no desenvolvimento de mudas de espécies florestais após a incorporação de pó de rocha fosfatada no solo, objetivou-se avaliar o desenvolvimento de mudas de Schizolobium parahyba var. amazonicum em diferentes composições de pó de rocha Gnaisse, como fertilizante alternativo.

\section{MATERIAIS E MÉTODOS}

$\mathrm{O}$ experimento foi realizado durante $\mathrm{o}$ período de setembro a novembro de 2018 no viveiro florestal da Universidade Federal do Tocantins, Campus de Gurupi-TO. A altitude da área experimental é de $287 \mathrm{~m}$, sob as coordenadas geográficas de latitude $11^{\circ} 43^{\prime} 45^{\prime}$, S e longitude $49^{\circ} 04^{\prime} 07^{\prime \prime}$ W. Segundo Köppen o 
clima da região é do tipo $\mathrm{AW}$, definido como tropical úmido com precipitação média anual entre $1.500 \mathrm{~mm}$ a $1.600 \mathrm{~mm}$ e temperatura média ao longo do ano entre $22^{\circ} \mathrm{C}$ e $28^{\circ} \mathrm{C}$ (FONSECA, 2017).

As sementes são oriundas da cidade de Dom Elizeu - PA e foram doadas a Universidade Federal do Tocantins, Campus Gurupi-TO por pesquisadores do CPP (Centro de Pesquisa do Paricá). Foi realizado o beneficiamento das sementes no Laboratório de Sementes Florestais descartando as que apresentavam injúrias ou deformações (FONSECA, 2017). Após o beneficiamento foi realizada a quebra de dormência das sementes de Paricá em água quente a $90^{\circ} \mathrm{C}$ por 5 minutos.

Tabela 1. Análise Quantitativa pelo Método de Rietveld do pó de rocha, oriundo de rocha Gnaisse coletado na jazida da empresa CBM Mineração LTDA, localizada na rodovia BR 242, KM 370, S/N - Zona rural de Peixe - TO.

\begin{tabular}{lc}
\hline \multicolumn{2}{c}{ Jazida 2 } \\
\hline Calcita & \% \\
Dolomita & 0,89 \\
Magnetita & $<$ L.Q \\
Rutilo & $<$ L.Q \\
Anatásio & $<$ L.Q \\
Apatita & $<$ L.Q \\
Quartzo & $<$ L.Q \\
Ilmenita & 37,62 \\
Hematita & $<$ L.Q \\
Biotita & 0,59 \\
Muscovita & 1,62 \\
Albita & $<$ L.Q \\
Microclínio & 30,35 \\
Clorita & 25,23 \\
(<L.Q) = Proporção abaixo do limite quantificável. & 2,61 \\
\end{tabular}

A semeadura foi realizada em sacos plásticos de $11 \mathrm{~cm}$ x $21 \mathrm{~cm}$ no qual foram preenchidos com cinco composições de pó de rocha e substrato comercial Tropstrato Florestal ${ }^{\circ}$, foram semeadas três sementes por recipiente. Por volta de 20 dias após a emergência (DAE), foi realizado o raleio, mantendo apenas uma plântula por recipiente, objetivando favorecer o desenvolvimento da muda.

O experimento foi inteiramente casualizado, composto por cinco tratamentos e quatro repetições, sendo cada repetição composta por 10 plântulas. Os tratamentos consistiram em: T1: 0\% pó de Rocha (PDR) + $100 \%$ Substrato comercial, T2: $25 \%$ PDR + 75\% Substrato comercial, T3: 50\% PDR + 50\% Substrato comercial, T4: $75 \%$ PDR $+25 \%$ Substrato comercial e T5: $100 \%$ PDR $+0 \%$ Substrato comercial, após o plantio foram realizadas duas irrigações diárias utilizando regadores manuais.

Os parâmetros morfológicos escolhidos para avaliar o desenvolvimento das mudas, em resposta aos tratamentos com pó de rocha Gnaisse (fosfatada) no substrato, foram: porcentagem de emergência (E\%), obtido através da fórmula porcentagem de sementes emergidas, utilizando a fórmula: $\% \mathrm{E}=(\mathrm{N} / \mathrm{TS})$ $\mathrm{x} 100$, onde: $\% \mathrm{E}=$ percentual de sementes emergidas; $\mathrm{N}=$ número total de sementes emergidas, $\mathrm{TS}=$ número total de sementes. Índice de velocidade de emergência (IVE), utilizando a fórmula IVE $=\mathrm{E} 1 / \mathrm{N} 1+\mathrm{E} 2 / \mathrm{N} 2+\ldots$ $\mathrm{En} / \mathrm{Nn}$, onde IVE = índice de velocidade de emergência, E1, E2, En = número de plantas normais computadas na primeira contagem, segunda e última contagem. $\mathrm{N} 1, \mathrm{~N} 2, \mathrm{Nn}=$ número de dias da semeadura à primeira, 
segunda e última contagem. Altura da parte aérea $(\mathrm{H})$, diâmetro do coleto (DC), massa seca da parte aérea (MSPA) massa seca radicular (MSR) e massa seca total (MST).

A altura da parte aérea foi determinada aos 30, 45 e 60 dias após a emergência (DAE) a partir do nível do substrato até a ponta da última folha, utilizando-se régua graduada em centímetros; para os diâmetros do coleto realizou-se a medida no nível do substrato, utilizando-se paquímetro digital.

Para obtenção das variáveis MSPA e da MSR as plântulas foram retiradas dos sacos plásticos, e cuidadosamente desfizeram-se os torrões, retirando assim o excesso de substrato, cortadas, separando em parte aérea e raiz, submetidas à secagem por um período de aproximadamente 72 horas, em estufa de circulação de ar a $70{ }^{\circ} \mathrm{C}$, em embalagens de papel. O material foi posterior pesado em balança analítica (precisão $0,001 \mathrm{~g}$ ).

A massa seca total (MST) foi obtido pela soma das massas citadas e as relações entre as características medidas foram determinadas pela simples divisão entre elas, ressaltando que esses cálculos foram realizados para obter-se o índice de qualidade de Dickson (IQD). Este foi determinado em função da altura da parte aérea (H), do diâmetro do coleto (DC), da massa da parte aérea (MSPA) e da massa seca das raízes (MSR) por meio da fórmula (DICKSON et al., 1960) apresentada a seguir:

$$
\mathrm{IQD}=\frac{\mathrm{PMST}(\mathrm{g})}{\frac{H(\mathrm{~cm})}{D C(\mathrm{~cm})}+\frac{P M S P A(\mathrm{~g})}{P M S R(\mathrm{~g})}}
$$

Os dados foram submetidos à análise de variância e as médias, comparadas pelo teste de
Tukey, a 5\% de probabilidade, utilizando-se o programa estatístico Sisvar (FONSECA et al., 2017).

\section{RESULTADOS E DISCUSSÃO}

Os dados da análise de variância demonstram que não houve diferença significativa $(p>0,05)$ entre os tratamentos para nenhuma das variáveis analisadas, porcentagem de emergência (E\%), Índice de velocidade de emergência (IVE), Altura (H), Diâmetro do Coleto (DC), massa seca da Parte Aérea (MSPA), massa seca da Parte Radicular (MSR) e Índice de Qualidade Dickson (IQD). O tempo decorrido desde a semeadura até a emergência de todas as sementes foi de 11 dias, observando um maior percentual de emergência e IVE na combinação $25 \%$ PDR $+75 \%$ Substrato comercial sendo 93,75\%, 2,88, os valores respectivamente (Tabela 2 ).

Tabela 2. Percentual de Emergência e Índice de Velocidade de Emergência (IVE) de mudas aos 11 dias após a semeadura.

\begin{tabular}{ccc}
\hline TRATAMENTOS & EMERGÊNCIA \% & IVE \\
\hline 1 & $83.750 \mathrm{a}$ & $2.654 \mathrm{a}$ \\
2 & $93.750 \mathrm{a}$ & $2.885 \mathrm{a}$ \\
3 & $90.000 \mathrm{a}$ & $2.845 \mathrm{a}$ \\
4 & $82.500 \mathrm{a}$ & $2.452 \mathrm{a}$ \\
5 & $78.750 \mathrm{a}$ & $2.445 \mathrm{a}$ \\
\hline CV $(\%)$ & 14.69 & 12.55 \\
\hline Média & 85.750 & 2.654 \\
\hline
\end{tabular}

Médias de quatro repetições. Médias seguidas das mesmas letras não diferem entre si pelo teste Tukey a 5\% de probabilidade; T1: $0 \%$ pó de Rocha (PDR) + 100\% Substrato comercial, T2: $25 \%$ PDR + 75\% Substrato comercial, T3: 50\% PDR + 50\% Substrato comercial, T4: 75\% PDR + 25\% Substrato comercial e T5: $100 \%$ PDR + 0\% Substrato comercial.

Analisando as médias de alturas coletadas aos 30, 45 e 60 dias não apresentaram 
diferença significativa entre si. Suignard (2015) ao trabalhar com a mesma espécie estudada, avaliando as mesmas variáveis, encontrou resultados análogos aos deste trabalho.

Tabela 3. Altura $(\mathrm{cm})$ de mudas aos 30,45 e 60 dias após a germinação.

\begin{tabular}{cccc}
\hline \multirow{2}{*}{ TRATAMENTOS } & \multicolumn{3}{c}{ ALTURA (cm) } \\
\cline { 2 - 4 } & $\mathbf{3 0}$ & $\mathbf{4 5}$ & $\mathbf{6 0}$ \\
\hline 1 & $20.377 \mathrm{a}$ & $21.512 \mathrm{a}$ & $23.382 \mathrm{a}$ \\
2 & $19.140 \mathrm{a}$ & $19.835 \mathrm{a}$ & $22.347 \mathrm{a}$ \\
3 & $19.565 \mathrm{a}$ & $20.535 \mathrm{a}$ & $21.737 \mathrm{a}$ \\
4 & $19.307 \mathrm{a}$ & $20.192 \mathrm{a}$ & $21.460 \mathrm{a}$ \\
5 & $19.885 \mathrm{a}$ & $20.952 \mathrm{a}$ & $24.060 \mathrm{a}$ \\
\hline CV $(\%)$ & 6.59 & 7.32 & 5.60 \\
\hline Média & 19.65 & 20.60 & 22.60 \\
\hline
\end{tabular}

Médias de quatro repetições. Médias seguidas das mesmas letras não diferem entre si pelo teste Tukey a 5\% de probabilidade; T1: $0 \%$ pó de Rocha (PDR) $+100 \%$ Substrato comercial, T2: $25 \%$ PDR $+75 \%$ Substrato comercial, T3: 50\% PDR + 50\% Substrato comercial, T4: 75\% PDR + 25\% Substrato comercial e T5: $100 \%$ PDR + 0\% Substrato comercial.

Para a variável diâmetro do coleto os resultados não apresentaram diferença significativa entre os tratamentos ao longo dos 60 dias de avaliação do experimento. $\mathrm{O}$ tratamento que obteve a maior média nas medidas de 30, 45 e 60 dias foi o Tratamento 2 (25\% PDR $+75 \%$ Substrato comercial) com médias 3,84mm; 4,14mm e $4,53 \mathrm{~mm}$ respectivamente (Tabela 4). Comparando com estudos feitos em Eucalyptus grandis W. Hill ex Maiden por Knapik, (2005) com pó de Basalto, no qual observou que embora o tratamento 3 (20\% PB) tenha apresentado valor médio maior para diâmetro do coleto $(2,46$ $\mathrm{mm})$, não existiu diferença significativa entre os diâmetros médios das mudas obtidas com os tratamentos 3 e 2, com 20 e $10 \%$ PB, na composição do substrato respectivamente.
Tabela 4. Diâmetro do coleto (mm) de mudas aos 30, 45 e 60 dias após a germinação.

\begin{tabular}{cccc} 
& \multicolumn{4}{c}{ DC $(\mathbf{m m})$} \\
\cline { 2 - 4 } 1 & 30 & $\mathbf{4 5}$ & $\mathbf{6 0}$ \\
\hline 2 & $3.605 \mathrm{a}$ & $3.860 \mathrm{a}$ & $4.330 \mathrm{a}$ \\
& $3.842 \mathrm{a}$ & $4.142 \mathrm{a}$ & $4.530 \mathrm{a}$ \\
3 & $3.697 \mathrm{a}$ & $4.015 \mathrm{a}$ & $4.265 \mathrm{a}$ \\
4 & $3.787 \mathrm{a}$ & $4.137 \mathrm{a}$ & $4.257 \mathrm{a}$ \\
& & & \\
5 & $3.767 \mathrm{a}$ & $4.085 \mathrm{a}$ & $4.507 \mathrm{a}$ \\
\hline & & & \\
CV $(\%)$ & 4.75 & 3.24 & 3.73 \\
\hline & & & \\
Média & 3.740 & 4.048 & 4.378 \\
\hline
\end{tabular}

Médias de quatro repetições. Médias seguidas das mesmas letras não diferem entre si pelo teste Tukey a 5\% de probabilidade; T1: $0 \%$ pó de Rocha (PDR) $+100 \%$ Substrato comercial, T2: $25 \%$ PDR $+75 \%$ Substrato comercial, T3: 50\% PDR $+50 \%$ Substrato comercial, T4: $75 \%$ PDR $+25 \%$ Substrato comercial e T5: $100 \%$ PDR + 0\% Substrato comercial.

Os dados de massa seca da parte aérea e radicular obtiveram médias não significativas entre si, com o Tratamento 3 (50\% PDR $+50 \%$ substrato comercial) obtendo média de $1,507 \mathrm{~g}$ para massa seca da parte aérea. Já a massa seca da parte radicular o Tratamento 2 (25\% PDR + $75 \%$ substrato comercial) obteve a maior média 0,630g (Tabela 5). Observando um comportamento semelhante aos dados obtidos na variável morfológica altura, uma vez que existe relação direta entre as mesmas.

As médias gerais para os valores do Índice de Qualidade Dickson variaram entre 0,237 a 0,285 . Sendo que o tratamento que obteve a maior média foi o Tratamento 2 (25\% PDR $+75 \%$ substrato comercial) 0,285 , porém 
não houve diferença estatística entre os tratamentos.

Tabela 5. Massa seca da Parte Aérea; massa seca da Parte Radicular e massa Seca total

\begin{tabular}{|c|c|c|c|}
\hline \multirow{2}{*}{$\begin{array}{c}\text { TRATAMENTO } \\
\mathbf{S}\end{array}$} & \multicolumn{3}{|c|}{ MASSA SECA (g) } \\
\hline & PMSPA & PMSR & PMST \\
\hline 1 & $1.492 \mathrm{a}$ & $0.610 \mathrm{a}$ & $2.102 \mathrm{a}$ \\
\hline 2 & $1.440 \mathrm{a}$ & $0.630 \mathrm{a}$ & $2.070 \mathrm{a}$ \\
\hline 3 & $1.507 \mathrm{a}$ & $0.552 \mathrm{a}$ & $2.057 \mathrm{a}$ \\
\hline 4 & $1.412 \mathrm{a}$ & $0.487 \mathrm{a}$ & $1.900 \mathrm{a}$ \\
\hline 5 & $1.500 \mathrm{a}$ & $0.612 \mathrm{a}$ & $2.115 \mathrm{a}$ \\
\hline $\mathrm{CV}(\%)$ & 9.82 & 15.07 & 10.70 \\
\hline Média & 1.470 & 0.578 & 2.049 \\
\hline
\end{tabular}

Médias de quatro repetições. Médias seguidas das mesmas letras não diferem entre si pelo teste Tukey a 5\% de probabilidade; T1: 0\% pó de Rocha (PDR) $+100 \%$ Substrato comercial, T2: $25 \%$ PDR $+75 \%$ Substrato comercial, T3: 50\% PDR + 50\% Substrato comercial, T4: 75\% PDR + 25\% Substrato comercial e T5: $100 \%$ PDR + 0\% Substrato comercial.

Segundo Fonseca et al. (2002) e Delarmelina et al. (2015) o IQD é um bom indicador da qualidade das mudas, pois no seu cálculo são consideradas as robustezes e o equilíbrio da distribuição da biomassa na muda, ponderando os resultados de vários parâmetros importantes empregados para avaliação da qualidade. Entretanto, quando comparados os valores do IQD presente neste trabalho com os valores propostos por Hunt (1990) que estabelece valor mínimo de 0,20, concluímos que todos os tratamentos testados obtiveram médias superiores ao valor estabelecido por Hunt, demonstrando que as mudas estavam aptas a serem plantadas em campo (Tabela 6).
Tabela 6. Índice de Qualidade de Dickson de mudas Schizolobium parahyba var. amazonicum (Huber exDucke) Barneby.

\begin{tabular}{|c|c|}
\hline $\begin{array}{l}\text { TRATAMEN } \\
\text { TOS } \\
\end{array}$ & $\begin{array}{l}\text { ÍNDICE QUALIDADE } \\
\text { DICKSON (IQD) }\end{array}$ \\
\hline 1 & $0.270 \mathrm{a}$ \\
\hline 2 & $0.285 \mathrm{a}$ \\
\hline 3 & $0.267 \mathrm{a}$ \\
\hline 4 & $0.237 \mathrm{a}$ \\
\hline 5 & $0.272 \mathrm{a}$ \\
\hline $\mathrm{CV}(\%)$ & 13.88 \\
\hline Média & 0.266 \\
\hline \multicolumn{2}{|c|}{$\begin{array}{l}\text { Médias de quatro repetições. Médias seguidas das } \\
\text { mesmas letras não diferem entre si pelo teste Tukey } \\
\text { a 5\% de probabilidade; T1: } 0 \% \text { pó de Rocha (PDR) } \\
+100 \% \text { Substrato comercial, T2: } 25 \% \text { PDR }+75 \% \\
\text { Substrato comercial, T3: } 50 \% \text { PDR }+50 \% \text { Substrato } \\
\text { comercial, T4: } 75 \% \text { PDR + } 25 \% \text { Substrato comercial } \\
\text { e T5: } 100 \% \text { PDR + 0\% Substrato comercial. }\end{array}$} \\
\hline
\end{tabular}

\section{CONCLUSÃO}

Os resultados referentes a análise estatística evidenciaram que nenhum tratamento variou significativamente. Sugere que o pouco tempo de avaliação não foi suficiente para se ter resultados expressivos, uma vez que o pó de rocha libera nutrientes de forma lenta.

Todos os autores declararam não haver qualquer potencial conflito de interesses referente a este artigo.

\section{REFERÊNCIAS}

\begin{tabular}{llrr} 
ASSOCIAÇÃO & \multicolumn{2}{c}{ BRASILEIRA } & DE \\
PRODUTORES & DE & FLORESTAS \\
PLANTADAS. & Anuário & estatístico & da
\end{tabular}
ABRAF 2013 ano base 2012. Brasília, 148p, 2013.

DELARMELINA, W.M.; CALDEIRA, M.V.W.; FARIA, J.C.T.; LACERDA, L.C. Uso de resíduo orgânico em substrato para produção de Chamaecrista desvauxii (Collad.) Killip var. latistipula (Benth.). Cerne, v. 21, n. 1, p. 429437, 2015. 
DIAS, P.C.; ATAÍDE, G.M.; XAVIER, A.; OLIVEIRA, L. S.; PAIVA, H. N. Propagação vegetativa de Schilozobium amazonicum por estaquia. Cerne, v. 21, n. 3, p. 379-386, 2015.

FONSECA, É.P.; VALÉRI, S.V.; MIGLIORANZA, É.; FONSECA, N.A.N.; COUTO, L. Padrão de qualidade de mudas de Trema micrantha (L.) Blume, produzidas sob diferentes períodos de sombreamento. Revista Árvore, v. 26, n. 4, p. 515-523, 2002.

FONSECA E.F, SILVA G.O, TERRA D.L, SOUZA P.B. uso potencial da casca de arroz carbonizada na composição de substratos para produção de mudas de Anadenanthera peregrina (L) Speg. Revista Desafios - v. 04, n. 04, p. 32-40, 2017.

HUNT, G. A. Effect of styroblock design and Cooper treatment on morphology of conifer seedlings. In: Target seedling symposium, meeting of the western forest nursery associations, general technical REPORT rm200, 1990, Roseburg. Proceedings... Fort Collins: United States Departament of Agriculture, Forest Service, 1990. p. 218-222.

IWAKIRI, S.; ZELLER, F.; PINTO, J.A.; RAMIREZ, M.G.L.; SOUZA, M.M.; SEIXAS, R. Avaliação do potencial de utilização da madeira de Schilozobium amazonicum "Parica e Cecropia holeuca "Embaúba" para produção de painéis aglomerados. Revista Acta Amazônica, Manaus, v. 40, n. 2, p. 303-308, 2010.

KIEHL, E.J. Manual de Gema Composta: maturação e qualidade do composto. 3 . ed. Piracicaba: Kiehl EJ. 2002.

KNAPIK, J.G.; ÂNGELO, A.C. Pó de basalto e esterco equino na produção de mudas de Prunus sellowii koehne (Rosaceae). Floresta, v. 37, n.3, p.427-436, 2007.

KNAPIK, J.G. Utilização do pó de basalto como alternativa à adubação convencional na produção de mudas de Mimosa scabrella Benth. e Prunus sellowii Koehne. 2005. Dissertação (Mestrado), Curso de PósGraduação em Engenharia Florestal, Setor de Ciências Agrárias, Universidade Federal do Paraná, 2005.
MAEDA, S.; DEDECEK, R.A.; AGOSTINI, R.B.; ANDRADE, G.C.; SILVA, H.D. Pesquisa Florestal Brasileira, v. 54, n. 1, p. 97-104, 2007.

RESENDE, M.; CURI, N.; RESENDE, S.B.; CORRÊA, G. F. Pedologia: Base para distinção de ambientes. 4. Ed. Viçosa: Neput; 2002, 338 p.

STAPPE, J.L.; BALLONI, E.A. O uso da indústria de celulose como insumos na produção florestal. Revista IPEF, v. 40, n.1, p. 33-37, 1988.

SOL SUIGNARD, Y. Emergência e crescimento de mudas de Schilozobium parahyba var. amazonicum (Huber $x$ Ducke) Barney em tubetes sob diferentes substratos. 2015. 31 f. Monografia (Graduação em Engenharia Florestal) Universidade Federal do Recôncavo da Bahia. Cruz das Almas, BA, 2015.

SUGUINO, E.; JACOMINI, A.E.; LAZARINI, A.P.; MARTINS, N.A.; FARIA, A.M.; PERDONÁ, M.J. Utilização do pó de basalto na agricultura. Pesquisa \& Tecnologia, v. 8, n. 2, p. 1-5, 2011.

THEODORO, S.H. Fertilização da terra pela terra: uma alternativa para a sustentabilidade do pequeno produtor rural. 2000. Tese (Centro de Desenvolvimento Sustentável) Universidade de Brasília; 2000.

THEODORO, S.H.; LEONARDOS, O.H. The use of rocks to improve family agriculture in Brazil. Anais... da Academia Brasileira de Ciências, v. 78, n. 721, 2006.

THOMAZ, L.B. CRESCIMENTO INICIAL DE ANGICO-VERMELHO (Anadenathera peregrina (L) SPEG.) EM DIFERENTES DOSES DE NPK. 2012. Monografia (Departamento de Ciências Florestais e da Madeira). Universidade Federal do Espírito Santo. Jerônimo Monteiro. 2012.

WOLSCHICK, P.R.D.; SCHUCH, F.S.; GELBER, T.; SARTORETTO, L.M. Efeito do pó de rocha basáltica sobre a germinação de Cedrela fissilis. Agropecuária Catarinense, Florianópolis, v.29, n.3, p.76-80, set./dez. 2016. 